\title{
Asymptotic normality of the lengths of a class of nonparametric confidence intervals for a regression parameter*
}

\author{
Madan L. PURI† and Tiee-Jian WU \\ Indiana University and University of Houston
}

Key words and phrases: Rank statistics, linear regression model, confidence intervals, asymptotic normality.

AMS 1980 subject classifications: Primary 62E20, 62G15, secondary $62 \mathrm{~J} 05$.

\begin{abstract}
In the linear regression model, the asymptotic distributions of certain functions of confidence bounds of a class of confidence intervals for the regression parameter are investigated. The class of confidence intervals we consider in this paper are based on the usual linear rank statistics (signed as well as unsigned). Under suitable assumptions, if the confidence intervals are based on the signed linear rank statistics, it is established that the lengths. properly normalized, of the confidence intervals converge in law to the standard normal distributions; if the confidence intervals are based on the unsigned linear rank statistics. it is then proved that a linear function of the confidence bounds converges in law to a normal distribution.
\end{abstract}

\section{RÉSUMÉ}

On aborde ici, dans le cadre d'un modèle de régression linéaire, le comportement de certaines fonctions des bornes d'une classe d'intervalles de confiance. La classe d'intervalles de confiance que nous considérons est construite à partir des statistiques de rangs linéaires habituelles. Sous certaines hypothèses, et en supposant que les intervalles de confiance sont basés sur des statistiques linéaires de rangs signés, on montre que les longueurs renormalisées des intervalles de confiance convergent en loi vers une distribution normale centrée-réduite. Si les intervalles de confiance proviennent de statistiques linéaires de rangs non-signés, on montre en outre qu une combinaison linéaire des bornes de confiance converge en loi vers une distribution normale.

\section{INTRODUCTION}

For each $N \geq 1$, let $Y_{N i}, 1 \leq i \leq N$, be independent random variables. Assume that

$$
Y_{N i}=X_{N i}+\Delta d_{N i}, \quad i=1, \ldots, N, \quad N=1,2, \ldots
$$

where $\Delta$ is an unknown real parameter in the interval $[-M, M]$ for some $M>0$, the $d_{N_{i}}$ 's are known real constants, and $X_{N i}, \ldots, X_{N N}$ are independent and identically distributed random variables with common unknown cumulative distribution function $F(x)$. We assume that $F \in \overline{\mathscr{F}}$, where

\footnotetext{
*The original. more detailed, version of this paper is available upon request to the authors.

tResearch supported by National Science Foundation Grant MCS 8301409
} 


$$
\begin{aligned}
\mathscr{F}= & \{F: F \text { is absolutely continuous, } \\
& f(x)=F^{\prime}(x) \text { is absolutely continuous, and } \\
& F \text { has finite Fisher's information, i.e. } \\
& \left.I(f)=\int_{-x}^{x}\left[\frac{f^{\prime}(x)}{f(x)}\right]^{2} f(x) d x<\infty\right\} .
\end{aligned}
$$

The confidence intervals of the paramter $\Delta$ we shall study are based on the linear rank statistics

$$
S_{N}^{+}(\mathbf{Y})=\sum_{i=1}^{N} c_{N i} \phi\left(\frac{R_{N_{i}}^{+}}{N+1}\right) \operatorname{sgn}\left(Y_{N i}\right)
$$

and

$$
S_{N}(\mathbf{Y})=\sum_{i=1}^{N} c_{N i} \phi\left(\frac{R_{N i}}{N+1}\right)
$$

respectively, where $\mathbf{Y}=\mathbf{X}+\Delta \mathbf{d}$ denotes the $N$-dimensional random vector $\left(Y_{N 1}, Y_{N 2}, \ldots, Y_{N N}\right)$. [In our study, we shall use the customary notation $\mathbf{V}$ for the vector $\left.\left(V_{N 1}, V_{N 2}, \ldots, V_{N N}\right)\right]$; the $c_{N i}$ 's are known constants; $R_{N i}^{+}$is the rank of $\left|Y_{N i}\right|$ among $\left|Y_{N 1}\right|$, $\left|Y_{N 2}\right|, \ldots,\left|Y_{N N}\right| ; R_{N i}$ is the rank of $Y_{N i}$ among $Y_{N 1}, Y_{N 2}, \ldots, Y_{N N} ; \operatorname{sgn}(y)=1$ or -1 according as $y \geq 0$ or $<0$; and $\phi$ (the score-generating function) satisfies the following conditions:

$$
\begin{aligned}
& \phi \text { is nonnegative, nonconstant and nondecreasing on }[0,1] \\
& \phi(0)=0, \\
& \phi^{\prime \prime} \text { is absolutely continuous, } \\
& \int_{0}^{1}\left[\phi^{\prime \prime \prime}(u)\right]^{2} d u<\infty
\end{aligned}
$$

Now, let $\Phi$ denote the cumulative distribution function of the standard normal distribution. For any $\alpha$ with $0<\alpha<1$ we shall consider the confidence sets of $\Delta$ of the forms

$$
D_{N}^{+}=\left\{t:\left|S_{N}^{+}(\mathbf{Y}-t \mathbf{d})\right| \leq u_{c}^{+}\right\}
$$

and

$$
D_{N}=\left\{t:\left|S_{N}(\mathbf{Y}-t \mathbf{d})\right| \leq u_{\mathbf{\alpha}}\right\},
$$

where

$$
u_{\alpha}^{+}=\left[\int_{0}^{1} \phi^{2}(u) d u\right]^{\frac{1}{2}} \Phi^{-1}\left(\frac{\alpha+1}{2}\right)
$$

and

$$
u_{\alpha}=\left[\int_{0}^{1}\{\phi(u)-\Phi\}^{2} d u\right]^{\frac{1}{2}} \Phi^{-1}\left(\frac{\alpha+1}{2}\right)
$$

with $\bar{\phi}=\int_{0}^{1} \phi(u) d u$.

In what follows, we shall use the symbols $P_{\Delta_{0}}$ and $P_{0}$ to mean that the probabilities are computed respectively for $\Delta=\Delta_{0}$ and $\Delta=0$ in (1.1). In Section 2, we shall prove that under proper assumptions on the $c_{N i}$ 's, the $d_{N i}$ 's and $F$ (symmetric) the confidence sets (1.6) are actually confidence intervals such that the lengths converge in $P_{\Delta_{0}}$ probability to some constants for any $\Delta_{0} \in[-M, M]$; furthermore, after suitable normalization, they 
converge in distribution to the standard normal one. Similar results will be derived in Section 3 for the confidence sets (1.7), with the exception that a linear function of the confidence bounds is proved to be asymptotically normal. (This result generalizes the one obtained by Jurečková (1973), in which the statistic $S_{N}(\mathbf{Y})$ defined by (1.4) is of Wilcoxon type [i.e., $\phi(u)=u$ in (1.4)] and $\mathbf{c}=\mathbf{d}$.) Our methods are mainly adaptations of the ideas of Antille (1972), van Eeden (1972), and Jurečková (1973).

\section{CONFIDENCE INTERVALS BASED ON THE SIGNED LINEAR RANK STATISTICS}

In this section, in addition to the assumptions stated in Section I, we further assume that the distribution function $F$ is symmetric about zero.

We also make the following assumptions on the c's and $d$ 's:

$$
\begin{gathered}
\sum_{i=1}^{N} c_{N i}^{2}=1, \quad N \geq 1 ; \quad \lim _{N \rightarrow x} \max _{1 \leq 1 \leq N} c_{N_{i}}^{2}=0 ; \quad \sum_{i=1}^{N} d_{N i}=0, \quad N \geq 1 ; \\
\max _{1 \leq i \leq N}\left|d_{N i}\right|=O\left(N^{-\frac{1}{2}}\right) ; \quad \lim _{N \rightarrow x}\left(\sum_{i=1}^{N} c_{N i} d_{N i}\right)=\gamma>0 \\
c_{N i} d_{N i} \geq 0 \quad \text { for all } i=1,2, \ldots, N, \quad N=1,2, \ldots \\
\left(\left|c_{N i}\right|-\left|c_{N i}\right|\right)\left(\left|d_{N i}\right|-\left|d_{N i}\right|\right) \geq 0 \\
\text { for all } i, j=1,2, \ldots, N, \quad N=1,2, \ldots
\end{gathered}
$$

Then, we have the following

LeMma 2.1. Under the assumptions (1.1)-(1.3), (1.5), and (2.1)-(2.5), it holds true for any $\Delta_{0} \in[-M, M]$ and $\alpha \in(0,1)$ that

$$
\lim _{N \rightarrow \infty} P_{\Delta_{0}}\left[\Delta_{0} \in D_{N}^{\dagger}\right]=\alpha .
$$

Proof. From (1.3), (1.6), and (1.8), we have

$$
\begin{aligned}
P_{\Delta_{0}}\left[\Delta_{0} \in D_{N}^{\dagger}\right] & =P_{\Delta_{11}}\left[\left|S_{N}^{\dagger}\left(\mathbf{Y}-\Delta_{0} \mathbf{d}\right)\right| \leq u_{\alpha}^{\dagger}\right] \\
& =P_{0}\left[\left|S_{N}^{\dagger}(\mathbf{Y})\right| \leq u_{\alpha}^{\dagger}\right] \\
& \rightarrow 2\left\{\Phi\left(u_{\alpha}\left[\int_{0}^{1} \phi^{2}(u) d u\right]^{-\frac{1}{2}}\right)-\Phi(0)\right\}=\alpha,
\end{aligned}
$$

where the convergence follows from Theorem 17 of Hušková (1970) and Lemma V.1.6a of Hájek and Sídák (1967). Q.E.D.

Throughout this section, we shall use $F^{*}$ to denote the cumulative distribution function $\left|X_{N 1}\right|$. [Thus $F^{*}(x)=2 F(x)-1$ if $x \geq 0$, and $F^{*}(x)=0$ otherwise.]

Now let us denote

$$
\tilde{\Delta}_{N}^{-}=\sup \left\{t: S_{N}^{\dagger}(\mathbf{Y}-t \mathbf{d})>u_{\alpha}^{+}\right\}
$$

and

$$
\bar{\Delta}_{N}^{+}=\inf \left\{t: S_{N}^{+}(\mathbf{Y}-t \mathbf{d})<-u_{\alpha}^{+}\right\} .
$$

It follows from the assumptions of this section and Theorem 3.1 of van Eeden (1972) that 
the statistics $S_{N}^{t}(\mathbf{Y}-t \mathbf{d})$ is a nonincreasing step function of $t$ for fixed $\mathbf{Y}$ with probability I. Therefore, we have almost surely that

$$
D_{N}^{+}=\left(\tilde{\Delta}_{N}^{-}, \tilde{\Delta}_{N}^{\dagger}\right),
$$

and the confidence sets $D_{N}^{+}$defined by (1.6) are actually confidence intervals.

The following lemma states that for any $\Delta_{0} \in[-M, M]$, the lengths of the above intervals converge to some constant in $P_{\Delta_{11}}$-probability.

LEMMA 2.2 Under the assumptions (1.1)-(1.3), (1.5), and (2.1)-(2.5), it holds true for any $\Delta_{0} \in[-M, M)$ that

$$
\left(\tilde{\Delta}_{N}^{+}-\tilde{\Delta}_{N}^{-}\right) \rightarrow B_{\alpha}^{+} \quad \text { in } P_{\Delta_{1}} \text {-probability, }
$$

where

$$
B_{\alpha}^{+}=u_{\alpha}^{+}\left(2 \gamma \int_{0}^{x} \phi^{\prime}\left(F^{*}(x)\right) f^{2}(x) d x\right)^{-1}
$$

and $u_{\alpha}^{\prime}$ is defined by (1.8).

The proof is given in the Appendix.

Let $W$ be a random variable with cumulative distribution function $F^{*}$. We define

$$
A_{N}^{2}=8 \sum_{i=1}^{N} c_{N i}^{2} d_{N i}^{2} B_{1}+\frac{8}{N}\left(\sum_{i-1}^{N} c_{N i} d_{N i}\right)^{2}\left(3 B_{1}+8 B_{2}+4 B_{3}\right),
$$

where $2 B_{1}=\operatorname{Var}\left[\phi^{\prime}\left(F^{*}(W)\right) f(W)\right], 2 B_{2}=\operatorname{Cov}\left[\phi^{\prime}\left(F^{*}(W)\right) f(W), \int_{W}^{x} \phi^{\prime \prime}\left(F^{*}(y)\right)\right.$ $\left.f^{2}(y) d y\right]$, and $2 B_{3}=\operatorname{Var}\left[\int_{W}^{x} \phi^{\prime \prime}\left(F^{*}(y)\right) f^{2}(y) d y\right]$.

THEOREM 2.1 Under the assumptions (1.1)-(1.3), (1.5), and (2.1)-(2.3), the random process

$$
\begin{aligned}
& \left\{\mathscr{L}_{N}^{* *}(\Delta)=A_{N}^{-1}\left(S_{N}^{1}(\mathbf{X}+\Delta \mathbf{d})-S_{N}^{1}(X)-4 \Delta \sum_{i=1}^{N} c_{N i} d_{N i} \int_{0}^{\infty} \phi^{\prime}\left(F^{*}(x)\right) f^{2}(x) d x\right):\right. \\
& \Delta \in[-C, C]\}
\end{aligned}
$$

converges weakly to the Gaussian process $\{\Delta Z: \Delta \in[-C, C]\}$, where $Z$ is a random variable having the standard normal distribution, and $C$ is an arbitrary positive real number.

For proof of this theorem, see Puri and Wu (1984).

Remark 2.1. By using the Cauchy-Schwarz inequality, it can be readily shown that $A_{N}{ }^{2}=O(N)$ under the assumptions (1.2), (1.5), and (2.1)-(2.3).

Using the above theorem, we are able to prove the following lemma.

Lemma 2.3. Under assumptions (1.1)-(1.3), (1.5), and (2.1)-(2.5), we have for any real number $y$ that

$$
\begin{aligned}
P_{\Delta_{i}}\left\{A_{N}^{-1}\{\right. & S_{N}^{+}\left\{\mathbf{Y}-\left(\tilde{\Delta}_{N}^{-}+\frac{1}{N}\right) \mathbf{d}\right\}-S_{N}\left\{\mathbf{Y}-\left(\tilde{\Delta}_{N}^{+}-\frac{1}{N}\right) \mathbf{d}\right\} \\
& \left.\left.-4 \sum_{i-1}^{N} c_{N i} d_{N i}\left(\tilde{\Delta}_{N}^{+}-\tilde{\Delta}_{N}^{-}\right) \int_{0}^{x} \phi^{\prime}\left(F^{*}(x)\right) f^{2}(x) d x\right] \leq y\right\} \\
\rightarrow & \Phi\left(\frac{y}{B_{a}^{1}}\right)
\end{aligned}
$$


and

$$
\begin{aligned}
P_{\Delta_{0}}\left\{A_{N}^{-1}[\right. & S_{N}^{+}\left\{\mathbf{Y}-\left(\tilde{\Delta}_{N}^{-}-\frac{1}{N}\right) \mathbf{d}\right\}-S_{N}^{+}\left\{\mathbf{Y}-\left(\tilde{\Delta}_{N}^{\dagger}+\frac{1}{N}\right\} \mathbf{d}\right) \\
& \left.\left.-4 \sum_{i=1}^{N} c_{N i}(x) d_{N i}\left(\tilde{\Delta}_{N}^{+}-\tilde{\Delta}_{N}^{-}\right) \int_{0}^{x} \phi^{\prime}\left(F^{*}(x)\right) f^{\prime}(x) d x\right] \leq y\right\} \\
& \rightarrow \Phi\left(\frac{y}{B_{\alpha}^{+}}\right), \quad \text { (ii) }
\end{aligned}
$$

where $B_{\alpha}^{\prime}>0$ is defined in (2.9) and $A_{N}$ is defined in (2.10).

Proof. We first prove (i). Let us define for $N=1,2, \ldots$

$$
T_{N}^{+}= \begin{cases}\left(\tilde{\Delta}_{N}^{+}-\tilde{\Delta}_{N}^{-}-\frac{2}{N}\right)^{-1} & \text { if }\left(\tilde{\Delta}_{N}^{\prime}-\tilde{\Delta}_{N}^{-}-\frac{2}{N}\right) \neq 0, \\ 0 & \text { otherwise }\end{cases}
$$

It follows from Lemma 2.2 that

$$
T_{N}^{1} \rightarrow\left(B_{n}^{1}\right)^{-1} \quad \text { in } P_{\Delta_{1}} \text {-probability. }
$$

We now proceed by using arguments similar to those in Theorem III of Antille (1972). More specifically, since $\tilde{\Delta}_{N}^{+}$and $\tilde{\Delta}_{N}^{-}$are bounded in $P_{\Delta_{0}}$-probability and $P_{\Delta_{1},}\left[T_{N}^{\prime}=0\right] \rightarrow$ 0 , there exists $M^{\prime}>0$ such that

$$
P_{\Delta_{01}}\left[\left|\tilde{\Delta}_{N}^{+}-\frac{1}{N}\right|>M^{\prime}\right]<\frac{\varepsilon}{3}, \quad P_{\Delta_{10}}\left[\left|\tilde{\Delta}_{N}^{-}+\frac{1}{N}\right|>M^{\prime}\right]<\frac{\varepsilon}{3}, \quad \text { and } \quad P_{\Delta_{01},}\left[T_{N}^{\circ}=0\right]<\frac{\varepsilon}{3}
$$

for all large $N$ and arbitrary $\varepsilon>0$. Therefore, for any real number $y$, we have

$$
\begin{aligned}
& P_{\Delta_{11}}\left\{T_{N}^{+}\left[\mathscr{L}_{N}^{*}\left(\Delta_{0}-\left(\bar{\Delta}_{N}^{-}+\frac{1}{N}\right)\right)-\mathscr{L}_{N}^{*}\left(\Delta_{0}-\left(\tilde{\Delta}_{N}^{+}-\frac{1}{N}\right)\right)\right] \leq y\right\} \\
& \leq P_{\Delta_{11}}\left\{T_{N}^{+}\left[\mathscr{L}_{N}^{*}\left(\Delta_{0}-\left(\tilde{\Delta}_{N}^{-}+\frac{1}{N}\right)\right)-\mathscr{L}_{N}^{*}\left(\Delta_{0}-\left(\tilde{\Delta}_{N}^{+}-\frac{1}{N}\right)\right)\right] \leq y_{N} T_{N}^{\dagger} \neq 0,\right. \\
& \left.\left|\tilde{\Delta}_{N}+\frac{1}{N}\right| \leq M^{\prime},\left|\tilde{\Delta}_{N}^{\prime}-\frac{1}{N}\right| \leq M^{\prime}\right\}+\varepsilon \\
& \leq P_{\left.\Delta_{1}\right)}\left\{\inf _{\substack{\leq s, t<M^{\prime} \\
j \neq t}}(s-t)^{-1}\left[\mathscr{L}_{N}^{*}\left(\Delta_{0}-t\right)-\mathscr{Y}_{N}^{*}\left(\Delta_{0}-s\right)\right] \leq y\right\}+\varepsilon
\end{aligned}
$$

for any arbitrary $\varepsilon>0$ and all large $N$. It now follows from (2.12) and Theorem 2.1 that

$$
\begin{aligned}
& \left.\varlimsup P_{\Delta_{0}}\left\{T_{N}^{+}\left[\mathscr{L}_{N}^{*}\left(\Delta_{0}-\left(\bar{\Delta}_{N}^{-}+\frac{1}{N}\right)\right)-\mathscr{L}_{N}^{*}\left(\Delta_{0}-\left(\tilde{\Delta}_{N}^{+}-\frac{1}{N}\right)\right)\right] \leq y\right]\right\} \\
& \leq P\left\{\inf _{\substack{M^{\prime} \leq . \leq M^{\prime} \\
s \neq 1}}(s-t)^{-1}\left[\left(\Delta_{0}-t\right) Z-\left(\Delta_{0}-s\right) Z\right] \leq y\right\} \\
& =P[Z \leq y] \text {. }
\end{aligned}
$$


where $Z \sim \mathbf{N}(0,1)$.

Similarly, we have

$$
\begin{aligned}
& P_{\Delta_{0}}\left\{T_{N}^{+}\left[\mathscr{L}_{N}^{*}\left(\Delta_{0}-\left(\tilde{\Delta}_{N}^{-}+\frac{1}{N}\right)\right)-\mathscr{L}_{N}^{*}\left(\Delta_{0}-\left(\tilde{\Delta}_{N}^{+}-\frac{1}{N}\right)\right)\right] \leq y\right\} \\
& \geq P_{\Delta_{0}}\left\{T_{N}^{+}\left[\mathscr{L}_{N}^{*}\left(\Delta_{0}-\left(\tilde{\Delta}_{N}^{-}+\frac{1}{N}\right)\right)-\mathscr{L}_{N}^{*}\left(\Delta_{0}-\left(\tilde{\Delta}_{N}^{+}-\frac{1}{N}\right)\right)\right] \leq y, T_{N}^{+} \neq 0,\right. \\
& \left.\geq\left|\tilde{\Delta}_{N}^{-}+\frac{1}{N}\right| \leq M^{\prime},\left|\tilde{\Delta}_{N}^{+}-\frac{1}{N}\right| \leq M^{\prime}\right\} \\
& \geq P_{\Delta_{1}}\left\{\sup _{\substack{M^{\prime} \leq s, t \leq M^{\prime} \\
i \neq 1}}(s-t)^{-1}\left[\mathscr{L}_{N}^{*}\left(\Delta_{0}-t\right)-\mathscr{L}_{N}^{*}\left(\Delta_{0}-s\right)\right] \leq y\right\} .
\end{aligned}
$$

Hence

$$
\begin{array}{r}
\underline{\lim } P_{\Delta_{i 1}}\left\{T_{N}^{+}\left[\mathscr{L}_{N}^{*}\left(\Delta_{0}-\left(\tilde{\Delta}_{N}^{-}+\frac{1}{N}\right)\right)-\mathscr{L}_{N}^{*}\left(\Delta_{\imath)}-\left(\tilde{\Delta}_{N}^{+}-\frac{1}{N}\right)\right)\right] \leq y\right\} \\
\geq P[Z \leq y] .
\end{array}
$$

(2.11), (2.13) and (2.14) imply

$$
\mathscr{L}_{N}^{*}\left(\Delta_{0}-\left(\tilde{\Delta}_{N}^{-}+\frac{1}{N}\right)\right)-\mathscr{E}_{N}^{*}\left(\Delta_{0}-\left(\tilde{\Delta}_{N}^{+}-\frac{1}{N}\right)\right) \rightarrow \mathrm{N}\left(0,\left(B_{\alpha}^{+}\right)^{2}\right)
$$

in distribution. (i) now follows from (2.15) and the fact that $8 A_{N}^{-1} N^{-1} \Sigma_{i=1}^{N} c_{N i} d_{N i} \int_{0}^{x}$ $\phi^{\prime}\left(F^{*}(x)\right) f^{2}(x) d x \rightarrow 0$ as $N \rightarrow \infty$, which follows from Remark 2.1 and (2.3).

(ii) can be proved similarly. Q.E.D.

The main result of this section is the following theorem.

THEOREM 2.2 Under the assumptions (1.1)-(1.3), (1.5), and (2.1)-(2.5),

$$
s_{N}^{-1}\left(\tilde{\Delta}_{N}^{+}-\tilde{\Delta}_{N}^{-}-a_{N}^{\dagger}\right) \rightarrow \mathrm{N}(0,1)
$$

in distribution, where $a_{N}^{+}=\gamma B_{u}^{+}\left(\sum_{i=1}^{N}\left(c_{N i} d_{N i}\right)^{-1}, s_{N}=\gamma A_{N}\left(B_{\alpha}^{+}\right)^{2}\left(2 \sum_{i=1}^{N} c_{N i} d_{N i} u_{\alpha}^{+}\right)^{-1}\right.$, and $u_{\alpha}^{+}, A_{N}$, and $B_{a x}^{+}$are defined by (1.8), (2.10), and (2.9), respectively.

Proof. From (4.11) (in the Appendix) and (i) of Lemma 2.3, we have

$$
\begin{array}{r}
\overline{\lim } P_{\Delta_{i}}\left\{A_{N}^{-1}\left[2 u_{\alpha}^{\prime}-4 \sum_{i=1}^{N} c_{N i} d_{N i}\left(\tilde{\Delta}_{N}^{\dagger}-\tilde{\Delta}_{N}^{-}\right) \int_{0}^{\infty} \phi^{\prime}\left(F^{*}(x)\right) f^{2}(x) d x\right] \leq y\right\} \\
\leq \Phi\left(\frac{y}{B_{\alpha}^{\dagger}}\right) .
\end{array}
$$

Similarly, from (4.10) (in the Appendix) and (ii) of Lemma 2.3,

$$
\begin{array}{r}
\left.\underline{\lim } P_{\Delta_{0}}\left\{A_{N}^{-1}\left[2 u_{a x}^{\prime}-4 \sum_{i=1}^{N} c_{N i} d_{N i}\left(\tilde{\Delta}_{N}^{+}-\tilde{\Delta}_{N}^{-}\right) \int_{0}^{x} \phi^{\prime} F^{*}(x)\right) f^{2}(x) d x\right] \leq y\right\} \\
\geq \Phi\left(\frac{y}{B_{\alpha}^{+}}\right)
\end{array}
$$

for any real number $y$. The proof follows as an immediate consequence of (2.9), (2.17), and (2.18). Q.E.D. 


\section{CONFIDENCE INTERVALS BASED ON THE UNSIGNED LINEAR RANK STATISTICS}

In this section, we do not need the assumption that $F$ is symmetric about zero. We make the following assumptions on the $c$ 's and $d$ 's:

$$
\begin{gathered}
\sum_{i=1}^{N} c_{N i}=\sum_{i=1}^{N} d_{N i}=0, \quad \sum_{i=1}^{N} c_{N i}^{2}=\sum_{i=1}^{N} d_{N i}^{2}=1, \quad N=2,3, \ldots, \\
\max _{1 \cdots i=N}\left|c_{N i}\right|=O\left(N^{-\frac{1}{2}}\right), \quad \max _{1 \rightarrow i \cdots N}\left|d_{N i}\right|=O\left(N^{-\frac{1}{2}}\right), \\
\lim _{N \rightarrow \infty} \sum_{i=1}^{N} c_{N i} d_{N i}=\gamma>0, \\
\left(c_{N i}-c_{N i}\right)\left(d_{N i}-d_{N i}\right) \geq 0 \quad \text { for } i, j=1,2, \ldots, N, \quad N=1,2, \ldots .
\end{gathered}
$$

The results of this section can be derived by using arguments similar to those in deriving the results in the previous section. Therefore, we shall not give too many details.

LEMMA 3.1. Under the assumptions $(1.1)-(1.2),(1.4)-(1.5)$, and $(3.1)-(3.4)$, it holds true for any $\Delta_{0} \in[-M, M]$ and $\alpha \in(0,1)$ that

$$
\lim _{N \rightarrow \infty} P_{\Delta_{11}}\left[\Delta_{0} \in D_{N}\right]=\alpha \text {. }
$$

Proof. Similar to the proof of Lemma 2.1 (replace $D_{N}^{\prime}$ by $D_{N}, S_{N}^{\prime}$ by $S_{N}$, and $u_{\alpha}^{\prime}$ by $u_{x}$ ). The convergence follows from Theorem V.I.6.a and Lemma V.I.6.a of Hájek and Sídák (1967). Q.E.D.

Denote

$$
\tilde{\Delta}_{N}^{\prime}=\sup \left\{t: S_{N}(\mathbf{Y}-t \mathbf{d})>u_{\mathrm{u}}\right\}
$$

and

$$
\tilde{\Delta}_{N}^{\prime \prime}=\inf \left\{t: S_{N}(\mathbf{Y}-t \mathbf{d})<-u_{\alpha}\right\} .
$$

It follows from the assumptions of this section and Theorem 2.1 of Jurečkova (1969) that with probability $I$ the statistics $S_{N}(\mathbf{Y}-t \mathbf{d})$ is a nonincreasing step function of $t$. Therefore

$$
D_{N}=\left(\bar{\Delta}_{N}^{\prime}, \tilde{\Delta}_{N}^{\prime \prime}\right)
$$

and the confidence sets $D_{N}$ defined by (1.7) are actually confidence intervals.

The lengths of the above confidence intervals converge to some constant in $P_{\Delta_{11}}$-probability for any $\Delta_{0} \in[-M, M]$.

LEMmA 3.2. Under the assumptions (1.1)-(1.2), (1.4)-(1.5), and (3.1)-(3.4), it holds true for any $\Delta_{0} \in[-M, M]$ that

$$
\left(\tilde{\Delta}_{N}^{\prime \prime}-\tilde{\Delta}_{N}^{\prime}\right) \rightarrow B_{i x} \text { in } P_{\Delta_{11}} \text {-probability, }
$$

where

$$
B_{\mathrm{u}}=2 u_{\mathrm{ux}}\left(\gamma \int_{-x}^{x} \phi^{\prime}(F(x)) f^{2}(x) d x\right)^{-1}
$$

and $u_{x}$ is defined by (1.9). 
Proof. By (3.1)-(3.3), and by Lemma V.1.6.a and Theorem V1.2.4 of Hájek and Sǐdák (1967), we can proceed as in $\left(2,10\right.$ ) (replace $\tilde{\Delta}_{N}^{-}$by $\tilde{\Delta}_{N}^{\prime}, S_{N}^{+}$by $S_{N}$, and $u_{\alpha}^{+}$by $u_{\alpha}$ ) to obtain that $\tilde{\Delta}_{N}^{\prime}$ is asymptotically normal with mean

$$
\Delta_{0}-\Phi^{-1}\left(\frac{\alpha+1}{2}\right)\left(\int_{0}^{1}\{\phi(u)-\bar{\phi}\}^{2} d u\right)^{\frac{1}{2}}\left(\gamma \int_{-x}^{x} \phi^{\prime}(F(x)) f^{2}(x) d x\right)^{-1}
$$

and variance

$$
\int_{0}^{1}\{\phi(u)-\bar{\phi}\}^{2} d u\left(\gamma \int_{-\infty}^{\infty} \phi^{\prime}(F(x)) f^{2}(x) d x\right)^{-2}
$$

It can similarly be shown that $\tilde{\Delta}_{N}^{u}$ is asymptotically normal with mean

$$
\Delta_{0}+\Phi^{-1}\left(\frac{\alpha+1}{2}\right)\left(\int_{0}^{1}\{\phi(u)-\bar{\phi}\}^{2} d u\right)^{\frac{1}{2}}\left(\gamma \int_{-x^{*}}^{x} \phi^{\prime}(F(x)) f^{2}(x) d x\right)^{-1}
$$

and variance (3.10). Thus $\tilde{\Delta}_{N}^{\prime}$ and $\tilde{\Delta}_{N}^{\prime \prime}$ are both bounded in probability. It follows from the assumptions of this lemma and Theorem 3.1 of Jurečková (1969) that for any $\varepsilon>0$

$$
\begin{array}{r}
\lim _{N \rightarrow \infty} P_{\Delta_{0}}\left[\mid S_{N}\left(\mathbf{Y}-\left(\tilde{\theta}_{N} \pm \frac{1}{N}\right) \mathbf{d}\right)-\right. \\
S_{N}\left(\mathbf{Y}-\Delta_{0} \mathbf{d}\right)+\left(\tilde{\boldsymbol{\theta}}_{N} \pm \frac{1}{N}-\Delta_{0}\right) \gamma \\
\left.\times \int_{-\infty}^{\infty} \phi^{\prime}(F(x)) f^{2}(x) d x \mid \geq \varepsilon\right]=0
\end{array}
$$

when $\tilde{\theta}_{N}=\tilde{\Delta}_{N}^{\prime}$ as well as $\tilde{\Delta}_{N}^{u}$. Equation (3.12) implies

$$
\begin{aligned}
& \left(\tilde{\Delta}_{N}^{\prime \prime}-\tilde{\Delta}_{N}^{\prime}\right) \gamma \int_{-x}^{\infty} \phi^{\prime}(F(x)) f^{2}(x) d x \\
& -\left\lceil S_{N}\left(\mathbf{Y}-\left(\tilde{\Delta}_{N}^{\prime}+(-1)^{i} \frac{1}{N}\right) \mathbf{d}\right)-S_{N}\left(\mathbf{Y}-\left(\tilde{\Delta}_{N}^{\prime \prime}+(-1)^{i+1} \frac{1}{N}\right) \mathbf{d}\right)\right] \rightarrow 0 \\
& \text { in } P_{\Delta_{11}} \text {-probability }
\end{aligned}
$$

for both $i=1$ and $i=2$. But (3.5) and (3.6) imply

$$
S_{N}\left(\mathbf{Y}-\left(\tilde{\Delta}_{N}^{\prime}-\frac{1}{N}\right) \mathbf{d}\right)-S_{N}\left(\mathbf{Y}-\left(\tilde{\Delta}_{N}^{\prime \prime}+\frac{1}{N}\right) \mathbf{d}\right) \geq 2 u_{\alpha x}
$$

and

$$
S_{N}\left(\mathbf{Y}-\left(\tilde{\Delta}_{N}^{\prime}+\frac{1}{N}\right) \mathbf{d}\right)-S_{N}\left(\mathbf{Y}-\left(\tilde{\Delta}_{N}^{\prime \prime}-\frac{1}{N}\right) \mathbf{d}\right) \leq 2 u_{\alpha}
$$

The proof follows from (3.13)-(3.15) immediately. Q.E.D.

Now let $X$ be a random variable with cumulative distribution function $F$. Denote

$$
\begin{gathered}
V_{1}=2 \phi^{\prime}(F(X)) f(X), \quad V_{2}=\int_{X}^{*} \phi^{\prime \prime}(F(y)) f^{2}(y) d y \\
E_{N}^{2}=\frac{1}{4} \sum_{i=1}^{N} c_{N i}^{2} d_{N i}^{2} \operatorname{Var}\left(V_{1}\right) \\
+\frac{1}{N}\left(\sum_{i=1}^{N} c_{N i} d_{N i}\right)^{2}\left[\frac{3}{4} \operatorname{Var}\left(V_{1}\right)+2 \operatorname{Cov}\left(V_{1}, V_{2}\right)+\operatorname{Var}\left(V_{2}\right)\right]
\end{gathered}
$$




$$
a_{N}=\sum_{i=1}^{N} c_{N i} d_{N i} \int_{-x}^{x} \phi^{\prime}(F(x)) f^{2}(x) d x
$$

and

$$
b_{N}=\frac{1}{4}\left(\sum_{i=1}^{N} c_{N i} d_{N i}^{2}\right) \int_{-,}^{x} \phi^{\prime \prime}(F(x)) f^{3}(x) d x
$$

THEOREM 3.1. Under the assumptions (1.1)-(1.2), (1.4)-(1.5), and (3.1)-(3.3), let $C$ be an arbitrary positive real number. Then the random process

$$
\left\{\mathscr{L}_{N}(\Delta)=E_{N}^{-1}\left[S_{N}(\mathbf{X}+\Delta \mathbf{d})-S_{N}(\mathbf{X})-\Delta a_{N}-\Delta^{2} b_{N}\right]: \Delta \in[-C, C]\right\}
$$

converges weakly to the Gaussian process $\{\Delta Z: \Delta \in[-C, C]\}$, where $Z \sim N(0,1)$.

For proof see Carlson (1982).

Remark 3.1. In view of $(1.2),(1.5),(3.1)-(3.3)$, and the Cauchy-Schwarz inequality, it can easily be shown that $E_{N}^{-2}=O(N)$.

LEMMA 3.3. Under the assumptions (1.1)-(1.2), (1.4)-(1.5), and (3.1)-(3.4), it holds true for any real number $y$ and any $\Delta_{0} \in[-M, M)$ that

$$
\begin{array}{r}
P_{\Delta_{11}}\left\{E _ { N } ^ { - 1 } \left[S_{N}\left(\mathbf{Y}-\left(\tilde{\Delta}_{N}^{\prime}+(-1)^{i} \frac{1}{N}\right) \mathbf{d}\right)-S_{N}\left(\mathbf{Y}-\left(\tilde{\Delta}_{N}^{\prime \prime}+(-1)^{\prime \prime}+\frac{1}{N}\right) \mathbf{d}\right)\right.\right. \\
\left.\left.-\left(\tilde{\Delta}_{N}^{\prime \prime}-\bar{\Delta}_{N}^{\prime}\right) a_{N}+\left(\bar{\Delta}_{N}^{\prime \prime}+\tilde{\Delta}_{N}^{\prime}-2 \Delta_{0}\right) B_{\mathrm{a}} b_{N}\right] \leq y\right\} \rightarrow \Phi\left(\frac{y}{B_{\alpha}}\right)
\end{array}
$$

both for $i=0$ and $i=1$.

Proof. Let $i=0$. We define for $N=1,2, \ldots$

$$
T_{N}= \begin{cases}\left(\tilde{\Delta}_{N}^{\prime \prime}-\tilde{\Delta}_{N}^{\prime}-\frac{2}{N}\right)^{-1} & \text { if } \tilde{\Delta}_{N}^{u}-\tilde{\Delta}_{N}^{\prime}-\frac{2}{N} \neq 0, \\ 0 & \text { otherwise. }\end{cases}
$$

It follows from Lemma 3.2 that $T_{N} \rightarrow\left(B_{x}\right)^{-1}$ in $P_{\lambda_{1}}$-probability. Noting that $\tilde{\Delta}_{N}^{u}$ and $\tilde{\Delta}_{N}^{\prime}$ are bounded in $P_{\Delta_{11}}$-probability and using Theorem 3.1, we can proceed as in deriving $(2.12)-(2.15)$ (replace $T_{N}^{+}$by $T_{N}, \mathcal{L}_{N}^{*}$ by $\mathscr{L}_{N}, \tilde{\Delta}_{N}^{-}$by $\tilde{\Delta}_{N}^{\prime}$, and $\tilde{\Delta}_{N}^{+}$by $\tilde{\Delta}_{N}^{\prime \prime}$ ) to obtain

$$
\mathscr{L}_{N}\left(\Delta_{0}-\left(\tilde{\Delta}_{N}^{\prime}+\frac{1}{N}\right)\right)-\mathscr{L}_{N}\left(\Delta_{0}-\left(\tilde{\Delta}_{N}^{\prime \prime}-\frac{1}{N}\right)\right) \rightarrow \mathrm{N}\left(0, B_{a}^{2}\right)
$$

in distribution. But from (3.2), (3.18)-(3.19), and Remark 3.1, we have

$$
E_{N}^{-1} N^{-1} a_{N} \rightarrow 0 \text { and } E_{N}^{-1} b_{N}=O(1) \text {. }
$$

(3.21) follows immediately from (3.20), (3.22)-(3.23), and Lemma 3.2. Similarly, we can prove $(3.21)$ for the case $i=1$. The proof follows. Q.E.D.

The main result of this section is the following theorem.

THEOREM 3.2. If the assumptions (1.1)-(1.2), (1.4)-(1.5), and (3.1)-(3.4) are satisfied, then under $\Delta=\Delta_{0}$ it holds true that

$$
\left(E_{N} B_{u}\right)^{-1}\left[\left(\tilde{\Delta}_{N}^{\prime \prime}-\tilde{\Delta}_{N}^{\prime}\right) a_{N}-\left(\tilde{\Delta}_{N}^{\prime \prime}+\tilde{\Delta}_{N}^{\prime}\right) B_{u x} b_{N}-2\left(u_{\alpha}-\Delta_{0} B_{\alpha} b_{N}\right)\right] \rightarrow \mathrm{N}(0,1)
$$

in distribution for any $\Delta_{0} \in[-M, M]$, where $B_{\omega}, E_{N}, a_{N}$, and $b_{N}$ are defined by (3.8), 
(3.17), (3.18), and (3.19), respectively.

Proof. From (3.14), (3.15), and (3.21), we can proceed as in the proof of Theorem 2.2 to obtain the desired results. Q.E.D.

\section{APPENDIX}

Proof of Lemma 2.2. From the monotonicity of $S_{N}^{\prime}(\mathbf{Y}-t \mathbf{d})$ in $t$ we have for any real number $\delta$ that

$$
\begin{aligned}
& \lim _{N \rightarrow \infty} P_{\Delta_{u}}\left[\tilde{\Delta}_{N}^{-}>\delta\right]=\lim _{N \rightarrow \infty} P_{\Delta_{u}}\left[S_{N}^{\dagger}(\mathbf{Y}-\delta \mathbf{d})>u_{u}^{\dagger}\right] \\
& =\lim _{N \rightarrow \infty} P_{0}\left[S_{N}^{\dagger}\left(\mathbf{Y}+\left(\Delta_{0}-\delta\right) \mathbf{d}\right)>u_{\alpha}^{\dagger}\right] \\
& =1-\Phi\left\{\left(\int_{0}^{1} \phi^{2}(u) d u\right)^{-\frac{1}{2}}\left[u_{\alpha}^{1}-4\left(\Delta_{0}-\delta\right) \gamma \int_{0}^{\infty} \phi^{\prime}\left(F^{*}(x)\right) f^{2}(x) d x\right]\right\},
\end{aligned}
$$

where the last equality is from (2.3), Theorem 17 of Hušková ( 1970), and Lemma V.1.6a of Hájek and Sĩdák (1967). Equation (2.10) implies that $\bar{\Delta}_{N}^{-}$is asymptotically normal with mean

$$
\Delta_{0}-\Phi^{-1}\left(\frac{\alpha+1}{2}\right)\left(\int_{0}^{1} \phi^{2}(u) d u\right)^{\frac{1}{2}}\left(4 \gamma \int_{0}^{x} \phi^{\prime}\left(F^{*}(x)\right) f^{2}(x) d x\right)^{-1}
$$

and variance

$$
\left(\int_{0}^{1} \phi^{2}(u) d u\right)\left(4 \gamma \int_{0}^{*} \phi^{\prime}\left(F^{*}(x)\right) f^{2}(x) d x\right)^{-2}
$$

Similarly, $\tilde{\Delta}_{N}^{+}$is asymptotically normal with mean

$$
\Delta_{0}+\Phi^{\prime}\left(\frac{\alpha+1}{2}\right)\left(\int_{0}^{1} \phi^{2}(u) d u\right)^{\frac{1}{2}}\left(4 \gamma \int_{0}^{x} \phi^{\prime}\left(F^{*}(x)\right) f^{2}(x) d x\right)^{-1}
$$

and variance

$$
\left(\int_{0}^{1} \phi^{2}(u) d u\right)\left(4 \gamma \int_{0}^{*} \phi^{\prime}\left(F^{*}(x)\right) f^{2}(x) d x\right)^{-2} \text {. }
$$

Thus $\tilde{\Delta}_{N}^{-}$and $\tilde{\Delta}_{N}^{+}$are both bounded in probability, it then follows from the assumptions of this lemma and Theorem 3.2 of van Eeden (1972) that

$$
\begin{aligned}
\lim _{N \rightarrow x} P_{\Delta_{11}}\left[\mid S_{N}^{+}(\mathbf{Y}\right. & \left.-\left(\tilde{\Delta}_{N} \pm \frac{1}{N}\right) \mathbf{d}\right)-S_{N}^{\prime}\left(\mathbf{Y}-\Delta_{(1)} \mathbf{d}\right) \\
& \left.+4\left(\tilde{\Delta}_{N} \pm \frac{1}{N}-\Delta_{0}\right) \gamma \int_{0}^{x} \phi^{\prime}\left(F^{*}(x)\right) f^{2}(x) d x \mid \geq \varepsilon\right]=0
\end{aligned}
$$

and

$$
\begin{aligned}
\lim _{N \rightarrow \infty} P_{\Delta_{11}}\left[\mid S_{N}^{\dagger}(\mathbf{Y}\right. & \left.-\left(\tilde{\Delta}_{N}^{\prime} \pm \frac{1}{N}\right) \mathbf{d}\right)-S_{N}^{\dagger}\left(\mathbf{Y}-\Delta_{0} \mathbf{d}\right) \\
& \left.+4\left(\Delta_{N}^{+} \pm \frac{1}{N}-\Delta_{0}\right) \gamma \int_{0}^{x} \phi^{\prime}\left(F^{*}(x)\right) f^{2}(x) d x \mid \geq \varepsilon\right]=0
\end{aligned}
$$


for any $\varepsilon>0$. Equations (4.6) and (4.7) imply

$$
\begin{aligned}
4\left(\tilde{\Delta}_{N}^{+}-\tilde{\Delta}_{N}^{-}\right) \gamma \int_{0}^{x} \phi^{\prime}\left(F^{*}(x)\right) f^{2}(x) d x-\left[S_{N}^{\prime}\left(\mathbf{Y}-\left(\tilde{\Delta}_{N}^{-}-\frac{1}{N}\right) \mathbf{d}\right)\right. \\
\left.-S_{N}^{+}\left(\mathbf{Y}-\left(\tilde{\Delta}_{N}^{+}+\frac{1}{N}\right) \mathbf{d}\right)\right] \rightarrow 0 \quad \text { in } P_{\Delta_{0}} \text {-probability. }
\end{aligned}
$$

and

$$
\begin{aligned}
4\left(\tilde{\Delta}_{N}^{+}-\tilde{\Delta}_{N}^{-}\right) \gamma \int_{0}^{x} \phi^{\prime}\left(F^{*}(x)\right) f^{2}(x) d x-\left[S_{N}^{\dagger}\left(\mathbf{Y}-\left(\tilde{\Delta}_{N}^{-}+\frac{1}{N}\right) \mathbf{d}\right)\right. \\
\left.-S_{N}^{+}\left(\mathbf{Y}-\left(\tilde{\Delta}_{N}^{+}-\frac{1}{N}\right) \mathbf{d}\right)\right] \rightarrow 0 \quad \text { in } P_{\Delta_{4}} \text {-probability }
\end{aligned}
$$

But (2.6) and (2.7) imply

$$
S_{N}^{+}\left(\mathbf{Y}-\left(\tilde{\Delta}_{N}^{-}-\frac{1}{N}\right) \mathbf{d}\right)-S_{N}^{+}\left(\mathbf{Y}-\left(\tilde{\Delta}_{N}^{+}+\frac{1}{N}\right) \mathbf{d}\right) \geq 2 \mathbf{u}_{\alpha}^{+}
$$

and

$$
S_{N}^{\prime}\left(\mathbf{Y}-\left(\tilde{\Delta}_{N}^{-}+\frac{1}{N}\right) \mathbf{d}\right)-S_{N}^{\dagger}\left(\mathbf{Y}-\left(\tilde{\Delta}_{N}^{+}-\frac{1}{N}\right) \mathbf{d}\right) \leq 2 u_{\alpha \alpha}^{+}
$$

Combining (4.8) and (4.10) yields for any $\varepsilon>0$ that

$$
P_{\Delta_{01}}\left[4\left(\tilde{\Delta}_{N}^{\prime}-\tilde{\Delta}_{N}^{-}\right) \gamma \int_{0}^{x} \phi^{\prime}\left(F^{*}(x)\right) f^{2}(x) d x-2 u_{\alpha}^{+}<-\varepsilon\right] \rightarrow 0 .
$$

Similarly, combining (4.9) and (4.11) yields

$$
P_{\Delta_{1}}\left[4\left(\bar{\Delta}_{N}^{+}-\tilde{\Delta}_{N}^{-}\right) \gamma \int_{0}^{x} \phi^{\prime}\left(F^{*}(x)\right) f^{2}(x) d x-2 u_{\alpha}^{+}>\varepsilon\right] \rightarrow 0 .
$$

(4.12) and (4.13) lead to the desired result. Q.E.D.

\section{ACKNOWLEDGEMENT}

We would like to express our sincere thanks to the Associate Editor for suggestions which improved the presentation of the paper.

\section{REFERENCES}

Antille, A. (1972). Linearité asymptotique d'une statistique de rang. Z. Wahrsch. Verw. Gobiete, 24, 309-324. Billingsley. P. (1968). Convergence of Probability Measures. Wiley, New York.

Carlson. Mark A. (1982). Central limit problem for general rank-scores statistic process. Ph.D. Dissertation, Indiana University. Bloomington. Indiana.

Hájek, J. and Sídák. Z. (1967). Theory of Rank Tests. Academic Press, New York

Hušková, M. (1970). Asymptotic distribution of simple linear rank statistics for testing symmetry. $Z$. Wahrsch. Verw. Gebiete. 14, 308-322.

Jurečková. J. (1969). Asymptotic linearity of a rank statistic in regression parameter. Amn. Math. Statist., 40, $1889-1900$. 
Jurečková, J. (1973). Central limit theorem for Wilcoxon rank statistics process. Ann. Statist., 6, 1046-1060. Puri, M. L., and Wu, Tiee-Jian (1984). Gaussian approximation of signed linear rank statistics process. $J$. Statist. Plann. Inference (in press).

van Eeden, Constance (1972). An analogue, for signed rank statistics, of Jurečková's asymptotic linearity theorem for rank statistics. Ann. Math. Statist., 43, 791-802.

Recived 18 February 1983

Revised 28 February 1984

Accepted 20 March 1984
Department of Mathematics Swain Hall East

Indiana University

Bloomington, Indiana 47405

U.S.A.

Department of Mathematics

University of Houston

University Park

Houston, Teras 77004

U.S.A. 